\title{
Dexamethasone induced psychosis presenting with catatonic features
}

Catatonia is a complex condition characterized by the presence of various motor signs and symptoms. It remains a poorly understood, poorly studied, and poorly recognized syndrome. ${ }^{1}$ Catatonic features are seen in a range of psychiatric conditions including psychotic disorders, mood disorders, conversion disorder and dementia. ${ }^{2}$ Studies have also identified over 35 medical and neurological illnesses associated with catatonia including corticosteroids exposure, central nervous system (CNS) structural damage, encephalitis and other CNS infections, seizures, metabolic disturbances, phencyclidine exposure, neuroleptic exposure, lupus cerebritis, disulfuram, porphyria, and other conditions. These medical causes of catatonia account for between $20 \%$ to $30 \%$ of such cases. ${ }^{3}$

Whilst various authors have reported several cases of psychiatric illnesses such as mood disorders, psychotic disorders, delirium and anxiety disorders due to use of corticosteroids, there are however, few reported cases of catatonia due to steroids. A literature search revealed very few articles written on catatonia due to steroids. We report a case of apparent dexamethasone induced psychosis presenting with features of catatonia.

B. O, a 20year old female undergraduate in Nigeria, was brought to the hospital by her elder sister (who was the informant). She started using dexamethasone without prescription six months prior to presentation because she wanted to increase her body weight. She was introduced to the drug by the elder sister who was also using it for the same purpose. She initially started with a dose of $4 \mathrm{mg}$ daily (an unused sachet of the drug was brought to the hospital by the informant) but later increased this to $4 \mathrm{mg}$ twice daily about two months before presentation. Her reason for the increment was that she had observed that the drug causes insomnia and since her exams were approaching, she felt the need to take something that would keep her awake at night to study.

About two days before presentation, she was observed by the elder sister to have been staying awake almost throughout the night and was also behaving abnormally, for example trying to strip herself naked in public. She was also observed to be withdrawn and refusing to talk generally but at times murmuring the same words repeatedly. Based on these features, she was brought to the hospital. Detailed history from the informant did not reveal

Correspondence

Dr MU Dada

email: bu_dada@yahoo.com any abnormalities in the patient before she commenced the drugs and there was also no family history of any psychiatric illness. On admission, she was observed to be uncooperative as she refused to obey any instruction. She was also mute and maintained abnormal postures. Physical examination showed she had high grade fever, muscular rigidity (though some of these features may be suggestive of neuroleptic malignant syndrome, this patient however had no previous exposure to antipsychotics). She also had a copious whitish vaginal discharge and widespread hypo pigmented macular lesions on her body. Various laboratory tests done including a full blood count, electrolytes, urea and creatinine and retroviral screening did not reveal any abnormalities. Cortisol levels could however not be done due to unavailability of facilities to do this.

She was commenced on a parenteral benzodiazepine (diazepam) to which she responded favourably. She was later observed to be speaking irrelevantly with auditory and visual hallucinations, whilst in clear consciousness. Based on these psychotic symptoms, she was commenced on risperidone. She responded favourably to this and was discharged after two weeks on the ward, mentally stable with no evidence of thought disorder or hallucinations.

This case demonstrates that dexamethasone can precipitate psychotic symptoms with catatonic features (catatonic stupor) in previously well individuals. The prominent catatonic features seen in this patient were mutism, posturing, withdrawal, rigidity, immobility (assessed using the 14-item Bush-Francis catatonia screening instrument ${ }^{4}$ ). The evidence suggesting causation is that the patient was functioning well even while using the dexamethasone at the initial dose but shortly after she increased the dose the psychiatric complications began. Such causation has not been noted in the literature, with only one of such publications reporting a case of prednisolone induced mood disorder with catatonic features. ${ }^{5}$

Catatonia has been described by various authors as a"common functional final pathway" in expression of severe neuropsychiatric illness. ${ }^{6}$ Based on this, it is probable that the catatonia occurring due to use of corticosteroids is a severe manifestation of a possible dexamethasone induced psychotic disorder in this patient. Probable theories for this causation include:

- The report by various researchers such as Wolkowitz that high levels of glucocorticoids cause an increase in dopamine levels which consequently leads to various psychiatric consequences. ${ }^{7}$ The effect of these glucocorticoids have been shown to be mediated by the 
presence of glucocorticoid receptors (GR) in the brain which mediate feed back action of steroids. ${ }^{8}$

- That in catatonic patients, as Northoff hypothesizes, there is modulation of cortical and sub cortical structures due to various conditions. The resultant hyperdopaminergic mesolimbic system may attempt a restitutive down regulation of dopamine to ward off psychosis, thus affecting other systems and producing motor symptoms seen in catatonia via the nigrostriatal pathway. ${ }^{9}$ Hence, corticosteroids which have been known to affect cortical and sub cortical structures can invariably cause catatonic features.

The catatonic features observed in this patient were managed successfully with a parenteral benzodiazepine while the psychotic symptoms were managed with risperidone. The choice of benzodiazepines was due to the unavailability of ECT. Benzodiazepines have also been used successfully in the management of catatonia associated with a range of disorders. ${ }^{10} \mathrm{Also}$, the choice of risperidone in the management of psychosis due to corticosteroids has been reported. ${ }^{11}$

This case report illustrates that psychotic symptoms with catatonic features can be a complication of corticosteroid use with parenteral benzodiazepines and oral antipsychotics beneficial in the management of such presentations.

MU Dada, L Oluwole, A Obadeji, OA Ajayi University Teaching Hospital, Ado-Ekiti, Ekiti State,

\section{References}

1. Penland HR, Weder N, Tampi RR. The catatonic dilemma expanded. Annals of General Psychiatry 2006; 5(14):1-9.

2. Caroff SN, Mann SC, Campbell EC, Sullivan KA. Epidemiology. In Catatonia: from psychopathology to neurobiology. Edited by: Caroff SN, Mann SC, Francis A, Fricchione GL. Washington, DC: American Psychiatric publishing, 2004: 15-31.

3. Carroll BT, Goforth HW. Medical catatonia. In Catatonia: from psychopathology to neurobiology Edited by: Caroff SN, Mann SC, Francis A, Fricchione GL. Washington, DC: American Psychiatric Publishing, 2004:121-127.

4. Bush G, Fink M, Petrides G, Dowling F, Francis A. Catatonia, I: rating scale and standardized examination. Acta Psychiatr Scand 1996; 93:129-136.

5. Grigg JR. Prednisolone induced mood disorder with associated catatonia. Journal of Geriatric Psychiatry and Neurology 1989; 2(1): 41-44.

6. Northoff G. What catatonia can tell us about "top-down modulation": a neuropsychiatric hypothesis. Behav Brain Sci 2002; 25:555-604.

7. Wolkowitz O, Sutton M, Koulo M. Chronic corticosterone administration in rats: behavioural and biochemical evidence of increased central dopaminergic activity. Eur J Pharmacol 1996; 122: 329-338.

8. De Kloet ER, Reul JMH. Feedback action and tonic influence of corticosteroids on brain receptor systems arising from the heterogeneity of brain. Psychoneuroendo 1987; 12:83-105.

9. Northoff G, Kotter R, Baumgart F, Danos P, Boeker H, Schlagenhauf F, et al. Orbitofrontal cortical dysfunction in akinetic catatonia: a functional magnetic resonance imaging study during negative emotional stimulation. Schizophr Bull 2004; 30:405-427.

10. Weder ND, Muralee S, Penland H, Tampi RR. Catatonia: a review. Ann Clin Psychiatry. 2008; 20(2):97-107.

11. Karmer B, Cottingham E. Risperidone in the treatment of steroid induced psychosis. Journal of Child and Adolescent Psychopharmacology 1999; 9(4):315-316. 